\title{
Programa "DT-Tênis 60+": uma proposta de sistematização para o aprendizado do tênis e prevenção de quedas de idosos
}

\author{
"DT-Tennis 60+" Program: a proposal for systematizing the learning of tennis \\ and the prevention of falls among the elderly
}

Luciano Juchem, Marcelo de Maio Nascimento

Universidade Federal do Vale do São Francisco (Univasf), Petrolina/PE, Brasil

\section{HISTÓRICO DO ARTIGO \\ Recebido: 12 maio 2020 \\ Revisado: 10 junho 2020 \\ Aprovado: 13 junho 2020}

\section{PALAVRAS-CHAVE:}

Tênis; Envelhecimento; Funções Executivas; Quedas.

\section{KEYWORDS:}

Tennis; Aging; Executive Functions; Falls.

\section{RESUMO}

OBJETIVO: Apresentar a proposta para sistematização do programa de treinamento "DT-Tênis 60+" específico para o aprendizado dos fundamentos do Tênis e prevenção de queda de idosos.

MÉTODOS: Estudo qualitativo descritivo, que revisou a literatura sobre os temas envelhecimento humano e quedas, a metodologia Play and Stay, sugerida para o ensino do tênis e a metodologia Dual Task, adequada para o planejamento de exercícios à prevenção de quedas de idosos.

RESULTADOS: Foram apresentadas duas ilustrações sobre a base conceitual da proposta "DT-Tênis 60+", além de um modelo estrutural sobre as fases da aula, contendo organização dos conteúdos, sequência das atividades e tempo de duração. $O$ texto encerra com imagens sobre materiais didáticos a serem utilizado na aula, seguido por exercícios práticos para adaptação do aluno com a raquete e o ensino da técnica forehand.

CONCLUSÃO: O princípio metodológico da proposta de treinamento "DT-Tênis 60+" pode ser adotado por profissionais da área do tênis em suas práticas diárias com a população idosa para o desenvolvimento de habilidades motoras e cognitivas, bem como, à redução do risco de quedas.

\section{ABSTRACT}

OBJECTIVE: To present the proposal for the systematization of the training program "DT-Tennis $60+$ " specifically for learning the fundamentals of Tennis and preventing falls from older adults.

METHODS: Qualitative descriptive study, which reviewed the literature on the themes of human aging and falls, the Play and Stay methodology, suggested for the teaching of tennis, and the Dual Task methodology, suitable for planning exercises to prevent older adult falls.

RESULTS: Two illustrations were presented on the conceptual basis of the proposal "DT-Tennis 60+", in addition to a structural model on the phases of the class, containing the organization of the contents, sequence of activities and duration. The text ends with images about teaching materials to be used in class, followed by practical exercises to adapt the student with the racket and the teaching of the forehand technique.

CONCLUSION: The methodological principle of the training proposal "DT-Tennis $60+$ " can be adopted by tennis professionals in their daily practices with the older adult population for the development of motor and cognitive skills, as well as, the reduction of the risk of falls. 


\section{INTRODUÇÃo}

De acordo com a organização Mundial de Saúde quedas de idosos são um problema de saúde pública (KALACHE, 2007). O motivo disso, é que quedas são responsáveis por altas taxas de mortalidade, podendo causar limitações funcionais e até mesmo levar o idoso a óbito (BARROS; PEREIRA; WEILLER, 2016). A literatura especializada aponta que cerca de 28 a $35 \%$ das pessoas, com 65 anos ou mais, caem pelo menos uma vez ao ano (McMULLAN et al., 2018), sendo que a taxa aumenta para 32 a $42 \%$ entre idosos com 75 anos ou mais (MARANESI et al., 2016). Outra particularidade da questão é que o caso é mais comum entre as mulheres (KALACHE, 2007), porque, comparativamente, estão mais expostas que os homens a acidentes domésticos (KHANUJA et al., 2018).

Por estes motivos, é importante pensar em medidas de baixo custo, efetivas e atrativas que possam prevenir idosos de caírem (MONTERO-ODASSO; SPEECHLEY, 2018). Nesse contexto, a prática do tênis pode ser empregada na prevenção de quedas de idosos, embora, essa modalidade esportiva ainda seja restrita às parcelas de maior poder aquisitivo em nosso país (MUELLER; RODRIGUES, 2009). Por intermédio da prática do tênis é possível que idosos (idade $\geq 60$ anos) mantenham ou desenvolvam capacidades motoras essenciais à resolução das atividades de vida diária (AVD) (BAZELLO et al., 2016), dentre elas, salienta-se a coordenação, a propriocepção, o equilíbrio, a velocidade da marcha, a força de membros inferiores e a flexibilidade dos membros superiores (GROPPEL; DINUBILE, 2009). Outro ponto favorável à prática do tênis entre idosos é que a própria dinâmica da modalidade estimula o indivíduo para se manter em movimento durante um tempo considerável. Por essa razão, o tênis é capaz de aumentar a função cardiovascular e pulmonar do idoso, contribuindo para redução da gordura corporal, o risco do desenvolvimento da diabetes e de doenças cardiovasculares (PLUIM et al., 2007).

Destaca-se ainda como vantagem para idosos, os beneficios de ordem social e emocional (BAZELLO et al., 2016), pois o ambiente onde esta modalidade é realizada atrai indivíduos de todas as faixas etárias, incentivando a troca de informações e formação de vínculos sociais. Deste modo, quanto maior for o tempo de prática do tênis, maior é a percepção do tenista sobre a importância deste esporte para sua saúde (SILVA; MARINSFRIO; SPIEKER, 2014).

Conforme Da Silva, Caminha e Gomes (2013), a partir dos 60 anos de idade se inicia uma nova fase da vida que é marcada por alterações biopsicossocias, que exigem do indivíduo um trabalho constante para vencer os novos desafios. Por essa razão, segundo os autores, o envolvimento do idoso em grupos de convivência, principalmente naqueles que oferecem práticas corporais, pode auxiliá-lo na liberação de tensões acumuladas no cotidiano. Isso significa dizer que o convívio social com indivíduos da mesma faixa etária amplia as possibilidades para que o idoso troque informações com os companheiros e, consequente, transforme ou adquirira novos conhecimentos. Para Cachioni, Sathler e Batistoni (2012), a troca de informações entre idosos oportuniza a formação de novos valores capazes inclusive de elevar a imagem corporal e autoestima.

Em estudo observacional desenvolvido por Silva, Marins, Spieker (2014), na cidade de Pelotas (RS), com 18 indivíduos de ambos os sexos (60-71 anos), os autores constataram que com o passar do tempo, tenistas idosos percebiam, cada vez mais, os benefícios desta atividade física para a saúde. Além disso, os entrevistados destacaram que o hábito de conviver semanalmente com outros idosos oferecia sustentação à manutenção de vínculos sociais, algo indispensável à melhora da percepção do bem-estar.

A literatura especializada da área do tênis destacou os benefícios de sua prática entre idosos, como, por exemplo, aumento dos níveis de aptidão física (PLUIM et al., 2007), melhora da funcionalidade (HERNANDES; DE FRANÇA BARROS, 2004), contribuindo para realização das AVDs (JUCHEM; DA SILVA; DA SILVA, 2016), bem como, à promoção da capacidade cognitiva (CASERTA; YOUNG; JANELLE, 2007). Entretanto, ainda hoje, não há estudos que tenham abordado à relação do tênis junto à população idosa com foco na prevenção de quedas considerando o treinamento motor e cognitivo. De tal modo, esta lacuna na literatura indica um campo oportuno para o desenvolvimento de estudos que possam contribuir à gênese do conhecimento teórico e prático da prevenção de quedas de idosos na área do tênis.

Diante do exposto, o objetivo deste estudo é apresentar a proposta para sistematização do programa de treinamento "DT-Tênis 60+" específico para o aprendizado do tênis e prevenção de queda de idosos.

\section{MÉTOdos}

Trata-se de um estudo qualitativo do tipo descritivo. Inicialmente, desenvolveu-se uma revisão de literatura com o propósito de examinar estudos anteriores que abordaram (1) envelhecimento humano, (2) o ensino do tênis com idosos e (3) princípios do treinamento de dupla tarefa com foco na prevenção de queda de idosos. Conforme Lakatos e Marconi (2003), a investigação bibliográfica permite verificar o estado do problema (fenômeno) a ser pesquisado até o momento. Em ums segunds fase, com base nas metodologias Play and Stay e Dual Task Training, foi elaborada a proposta inicial à sistematização do referencial teórico e prático (exercícios físicos) do treinamento "DT-Tênis 60+". A metodologia Play and Stay foi criada pela International Tennis Federation (ITF, 2012) para fundamentar o ensino do tênis. Desde 2007, a Confederação Brasileira de Tênis (CBT) recomenda este método para iniciação esportiva da modalidade (CORTELA et al., 2012).

A segunda metodologia adotada foi o treinamento Dual Task (dupla tarefa), que consiste na execução de duas ou mais tarefas simultaneamente com o objetivo de causar uma sobrecarga de atenção para o indivíduo (SILSUPADOL et al., 2009). Um exemplo prático é quando o aluno idoso é solicitado a caminhar (tarefa principal) e resolver concomitantemente demandas que exijam sua atenção (tarefa secundária), como a contagem regressiva a partir de 100, aplicando a subtração de três em três números. $\mathrm{O}$ treinamento DT incide em uma técnica frequentemente aplicada nos programas de prevenção de queda de idosos, uma vez que a tarefa secundária também funciona como parâmetro para estimativa do risco de queda, o que é intitulado como paradigma de dupla tarefa (GHAl; GHAI; EFFENBERG, 2017). Por conseguinte, com o auxílio do referencial teórico Play and Stay e Dual Task Training foram criados esquemas ilustrativos para 
detalhar a essência teórica da proposta "DT-Tênis 60+". Para facilitar o entendimento da proposta o texto apresenta uma série de sequências de imagens sobre materiais didáticos que podem ser utilizados, acompanhado por figuras que detalham exemplos práticos sobre a execução dos exercícios.

\section{RESULTADOS}

O envelhecimento humano é um processo natural e irreversível, responsável por alterações de caráter biopsicossociais sobre o organismo, que irá dificultar a adaptação do indivíduo no meio em que vive (McHUGH; GIL, 2018). Em relação à capacidade funcional, o avanço da idade traz mudanças significativas para o desempenho do sistema neural e musculoesquelético, gerando déficits sobre o padrão da marcha, controle postural, reduzindo também os níveis de força, flexibilidade e coordenação motora (SWANK, 2005). Outro tipo de alteração sobrevinda do envelhecimento incide no déficit do desempenho do sistema visual, vestibular e somatosensorial (propriocepção) (KLEINER et al., 2011). Disfunções nesses sistemas podem reduzir significativamente a autonomia do indivíduo durante à resolução de suas AVDs, o que impacta significativamente sobre a percepção da qualidade de vida e bem-estar (GOUVEIA et al., 2018).

Estudos de metanálise (RAFFEGEAU et al., 2019) e revisão sistemática (KEARNEY et al., 2013) destacaram que o envelhecimento fisiológico repercute sobre o desempenho das funções cerebrais, causando déficit no processamento das informações. Com isso, as Funções Executivas (FE) são afetadas, gerando distúrbios durante a realização de ações cotidianas essenciais, como: planejamento de tarefas, inibição de respostas, capacidade para abstrair e processar informações, além da memória de trabalho (UEHARA et al., 2013). Logo, quando comparados com indivíduos jovens ou adultos, idosos apresentam déficits de desempenho em todas as funções necessárias à resolução das AVDs, além de indicarem risco para queda (KEARNEY et al., 2013). Vale ressaltar que em idade avançada, quedas são responsáveis por lesões, dores, dias de hospitalização, além de integrarem a estatística de óbito da população idosa mundial (ABREU et al., 2018).

Uma medida para a manutenção dos níveis de aptidão física dos idosos consiste na prática regular de exercícios físicos. A literatura especializada indicou diferentes tipos, destacando-se aeróbicos (BOUAZIZ et al., 2016), resistidos (SOUZA et al., 2014), Pilates (NASCIMENTO, 2018), Tai-Chi (HALL; MISZKO; WOLF, 2009) e hidroginástica (SILVA et al., 2017). Todas essas atividades pertencem ao grupo de exercícios intitulados como de tarefa simples (simple task), ou seja, aqueles que requerem a resolução de uma única tarefa, não determinando sobrecarga à cognição e, por conseguinte, melhora significativa para o desempenho das FE.

Por outro lado, existem os exercícios de dupla tarefa (dual task), que combinam duas ou mais ações simultaneamente. Esta técnica exige esforço considerável, ao mesmo tempo, do aparelho motor e da condição cognitiva (LIEBHERR et al., 2016). Nas últimas décadas, as pesquisas sobre a metodologia de treinamento DT foram intensificadas (SMITH; CUSACK; BLAKE, 2016), isso qualificou significativamente o entendimento sobre os efeitos do treinamento DT sobre o processo do envelhecimento humano (PLUMMER; IYIGÜN, 2018).
A justificativa para o uso da metodologia DT surgiu da observação das características das atividades diárias realizadas por idosos. As ações exigem, ininterruptamente e concomitante, o gerenciamento cognitivo de diferentes tarefas externas (UEHARA et al., 2013). Um exemplo prático disso seria cozinhar e conversar com alguém, atravessar a rua e observar o fluxo dos carros, refletindo o tempo todo sobre as compras que devem ser feitas no supermercado do outro lado da rua. Isso significa dizer que as interações motoras e cognitivas destacadas nos exemplos citados consistem no chamado desempenho de DT (WOLLESEN et al., 2017). A literatura salienta que idosos constatados com baixo desempenho de DT também apresentam déficit na avaliação do equilíbrio corporal e da marcha, o que provoca aumento do risco de queda (MENANT et al., 2014).

\section{Desenvolvimento das habilidades do idoso por meio da prática do tênis}

Devido às características do próprio jogo, o tênis exige do praticante idoso constante foco direcionado para as diversas posições no espaço que a bola (pequena e veloz) assume. Além disso, o tenista idoso deve apresentar um aprimorado controle corporal para se movimentar com velocidade e destreza. Nesse contexto, quem joga tênis também não pode perder a percepção da extensão do braço que segura a raquete, devendo ser capaz de perceber seu posicionamento no espaço.

Todos esses exemplos integram um trabalho inconsciente e estritamente elaborado realizado pelo cérebro do tenista, em íntima associação com o desempenho do sistema sensorial (propriocepção). Por meio da propriocepção, o cérebro é capaz de analisar e criar respostas para as dificuldades impostas ao tenista pela trajetória, velocidade, efeito e potência da bola enviada pelo adversário, ou seja, o tempo que a bola demora para chegar no seu lado da quadra (agente intrínseco), e as diferentes direções e efeitos durante o trajeto (agente extrínseco). Considerações desta ordem, permitem analisar o tênis em sentido fenomenológico, visto que todo este processo é experiencial. De forma geral, todas as informações acumuladas pelo tenista, independentemente de sua faixa etária, são incorporadas (MERLEAU-PONTY, 1999), reflexos de um corpo-vivido em determinado tempo e espaço propiciado pela prática do tênis. Logo, do ponto de vista fenomenológico as experiências criadas em aulas ou na partida de tênis se constituem em alicerce à formação da corporeidade do indivíduo (FRANCO; MENDES, 2015), neste caso estamos nos referindo ao "eu-idoso-tenista".

Ao tecer o enfoque experiencial (fenomenológico) sobre a prática do tênis em idade avançada, a atitude filosófica nos permite expandir a questão para outro ponto essencial da área do esporte e da prevenção de quedas: a imagem corporal e imagem do movimento. Por meio da prática do tênis é possível que idosos aprimorem sua percepção do próprio corpo, implicando na conscientização de seus segmentos principalmente durante a movimentação, algo fundamental à eficiência das AVDs (STINS et al., 2015). No tênis, a imagem corporal acontece pela execução dos fundamentos técnicos e também da constante regulação entre a movimentação de pernas executada em diferentes direções e velocidades para alcançar as bolas, assim como a necessária mobilidade do tronco para que esteja equilibrado na execução dos golpes. Uma justificativa para o ensino do tênis 
tanto como atividade de lazer, como metodologia à prevenção de quedas de idosos pode ser encontrada na fisiologia do enveIhecimento humano, que destaca o déficit do desempenho do sistema visual, vestibular e somatossensorial nos idosos (KLEINER et al., 2011; UEHARA et al., 2013). Logo, a prática do tênis serve como uma medida preventiva, de lazer (pela interação social) e desafiadora (devido à imprevisibilidade das ações do oponente), para estimular e manter o funcionamento adequado dos mecanismos responsáveis pela estabilização do corpo no espaço.

Durante exercícios educativos para o desenvolvimento dos fundamentos técnicos, durante os treinos e nas disputas das partidas, as informações sobre a distância e velocidade da bola são captadas pelo músculo ocular, enquanto o aparelho vestibular analisa e corrige os posicionamentos angulares do corpo (MONTERO-ODASSO; SPEECHLEY, 2018). Todos esses dados posturais são enviados ao sistema nervoso central (SNC), que analisa os fatos e emite comandos rápidos e eficientes às extremidades do corpo para que os ajustes posturais necessários sejam realizados, evitando assim tropeços e quedas (NASCIMENTO, 2019). Em idade avançada, o bom funcionamento dos sistemas visual, vestibular e proprioceptivo, entre outros, é determinante para evitar quedas, o que depende da manutenção do centro de gravidade sobre a base do corpo e de um padrão da marcha inalterado (PLUMMER; IYIGÜN, 2018).

A Figura 1 ilustra o processamento de interação das informações motoras e cognitivas durante uma aula de tênis, segundo a proposta de treinamento "DT-Tênis $60+$ ". A base para criação do modelo foi fundamentada em princípios fenomenológicos (MERLEAU-PONTY, 1999), psicomotores do aprendizado de habilidades da técnica do tênis (CULPIN, 2018) e do treinamento DT com foco na prevenção de quedas de idosos (SILSUPADOL et al., 2009; WOLLESEN et al., 2017).

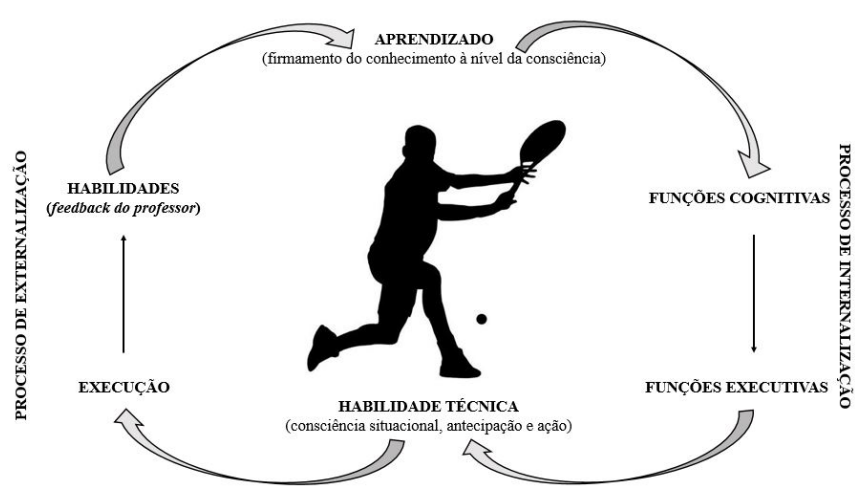

Figura 1. Modelo conceitual do treinamento de tênis para idosos "DT-Tênis 60+".

Fonte: Autores, 2019

Os procedimentos apresentados pelo modelo conceitual do treinamento "DT-Tênis $60+$ " se dividem basicamente em duas fases (Figura 1): internalização e externalização. A fase da internalização é composta pela percepção dos acontecimentos, isso inclui: i) captação dos dados posturais do próprio corpo e, ii) trajetória da bola. Esses procedimentos ocorrem com o auxílio dos órgãos sensoriais (visão, audição, propriocepção), em acordo com o SNC. Um exemplo prático é quando o idoso percebe que a bola se aproxima, diante disso, dados sensoriais são enviados "via aferente" ao cérebro que decide sobre quando e como o corpo deve (re)agir. Ainda na fase de internalização, há a ativação das funções executivas que são habilidades cognitivas essenciais à realização de ações complexas dirigidas para um determinado fim, como planejar, executar, sequenciar e monitorar ações (BANIQUED et al., 2018). Vale destacar que durante $o$ aprendizado da prática do tênis componentes das funções executivas como o raciocínio, a lógica, a estratégia, a tomada de decisões e a resolução de problemas são concomitantemente estimulados.

A fase da externalização contém, entre outros, a execução dos gestos motores. O momento é acompanhado por estímulos sequenciais processados nos lóbulos cerebrais (córtex pré-frontal dorsolateral e córtex cingulado anterior) (YOGEV-SELIGMANN; HAUSDORFF; GILADI, 2008). A prática do tênis exige ações rápidas e determinadas, sendo assim, a velocidade e eficácia das respostas exibidas pelo tenista idoso se apresentam diretamente proporcional à condição do seu desempenho "neurocognitivo" (KEARNEY et al., 2013). Isso significa dizer que, caso as funções executivas do idoso apresentem comprometimentos, é possível que a execução dos gestos motores ocorra com menor eficácia.

Nesse contexto, o SNC desenvolve um importante papel, uma vez que é encarregado de emitir "via eferente" informações às extremidades (membros), ajustando assim os gestos motores (MONTERO-ODASSO; SPEECHLEY, 2018). Vale salientar que é nesta fase que a técnica do tenista se consolida. Portanto, o arranjo entre as tentativas do aluno (acertos e erros) somado ao feedback do professor/treinador são determinantes à formação do "eu-tenista". Nesse processo, novos conhecimentos se conectam sucessivamente ao repertório de conhecimentos do tenista, consolidando-se dia a dia em sua memória (aprendizado).

\section{Treinamento "DT-Tênis 60+" na prática}

A prática do tênis exige a coordenação motora fina e grossa, o equilíbrio estático e dinâmico, bem como uma constante alteração do padrão da marcha, em acordo com a coordenação "olho-mão" (CASERTA; YOUNG; JANELLE, 2007). Por essas razões, é possível que idosos melhorem consideravelmente o desempenho de suas AVDs, visto que os procedimentos metodológicos do programa "DT-Tênis $60+$ " são direcionados à função inibitória de habilidades que exigem a troca simultânea de tarefas verbais e motoras, o que também ocorre mediante a variação da velocidade de processamento da memória de trabalho e perceptiva do idoso (CULPIN, 2018).

A Figura 2 apresenta os princípios conceituais do treinamento "DT-Tênis 60+". A estratégia que orienta o planejamento dos exercícios é a "prioridade variável", que conforme Silsupadol et al. (2009), é uma abordagem mais eficaz que a "prioridade fixa", pois nesta o foco da atenção é igual para as duas tarefas, em todos os momentos. Na "prioridade variável" os idosos são constantemente submetidos a um amplo e diversificado conjunto de tarefas cognitivas que alternam o foco de sua atenção durante à realização das tarefas de marcha e do equilíbrio estático e dinâmico. 
Na Figura 2, é possível visualizar as diretrizes para o planejamento da progressão do grau de complexidade das tarefas, que é o câmbio entre: i) diferentes bases de apoio e ii) procedimentos para a criação de estímulos cognitivos, próprios às FE.

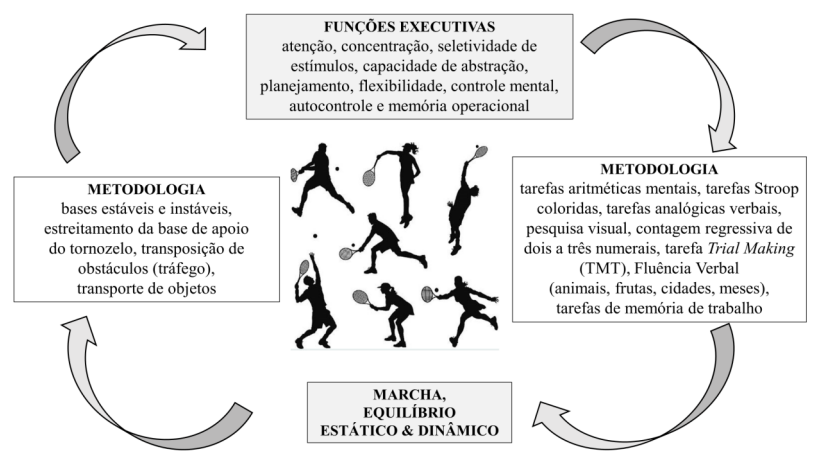

Figura 2. Diretrizes para a construção dos exercícios do treinamento "DT-Tênis 60+".

Fonte: Autores, 2019.

Estudo de revisão sistemática e metanálise (MENANT et al., 2014) mostrou que em se tratando da população idosa e da prevenção de quedas, a metodologia DT de "prioridade variável" é mais eficaz para o desempenho da velocidade da marcha, tanto na condição de tarefa simples (caminhar), como também para a marcha em condição de dupla tarefa (caminhar transportando objetos ou resolvendo tarefas cognitivas). Além disso, a metodologia DT de "prioridade variável" aprimora o controle do equilíbrio, reduzindo os níveis de oscilação corporal (LIEBHERR et al., 2016). Vale salientar que a metodologia de "prioridade variável" também é capaz de melhorar o desempenho cognitivo de idosos, diminuindo o tempo das respostas em testes cognitivos (GOUVEIA et al., 2018).

Na prática, o aumento do grau de complexidade dos exercícios do treinamento "DT-Tênis 60+" é determinado pela imposição realizada pela tarefa primária (motora) em combinação com a tarefa secundária (cognitiva). Outro ponto a considerar incide na habilidade motora e capacidade cognitiva de cada aluno. De tal modo, não se espera que o aluno atinja o nível mais elevado da complexidade de cada tarefa cognitiva ou motora, mas, sim que ele enfrente os desafios impostos pelas exigências criadas durante a aula de tênis.

Vale salientar que a progressão das tarefas é estabelecida a partir da observação diária do professor, portanto, deve existir ajuste entre os conteúdos e a evolução demonstrada pelo aluno. Aconselha-se que os níveis de complexidade dos exercícios e procedimentos progridam, no entanto, é recomendado manter o nível das exigências por três semanas e/ou 6 encontros. Após este período, sugere-se a troca dos exercícios e o aumento da complexidade das tarefas motoras e cognitivas. Desta maneira, o aluno tem tempo suficiente para se adaptar aos exercícios sem que o sistema neuromuscular, sensorial e cognitivo fique estagnado (LIEBHERR et al., 2016; GOUVEIA et al., 2018).

\section{Programa de exercícios "DT-Tênis 60+"}

A presente seção apresenta um conjunto de exercícios para a iniciação do tênis fundamentado no princípio "DT-Tênis 60+".
As atividades sugeridas são realizadas durante o aprendizado do tênis em situações que buscam o desenvolvimento e/ou a manutenção do desempenho motor e cognitivo, em tarefas de marcha e do controle postural. Os princípios do programa de treinamento "DT-Tênis 60+" consideraram as proposições da neurobiologia da queda de Fasano et al. (2012) e indicações Sherrington et al. (2017), específicas para o planejamento do treinamento DT à prevenção de quedas de idosos.

A proposta de sistematização das atividades do programa de treinamento do "DT-Tênis 60+" associa os fundamentos básicos desenvolvidos na iniciação do tênis com os princípios do treinamento de prevenção de quedas. Sendo assim, uma seção de "DT-Tênis 60+" inclui, por exemplo: (1) adaptação do aluno com a bola, com a raquete e com a raquete e a bola; 2) exercícios de forehand, 3) exercícios de backhand. Tudo isso é associado com: (1) necessidades motoras básicas para que o idoso execute suas AVDs com segurança, como, por exemplo: a) caminhada em diferentes superfícies; b) desvio de percurso e amplitude da passada, c) giros sobre o eixo, d) permanência na posição ereta sobre uma perna (tandem).

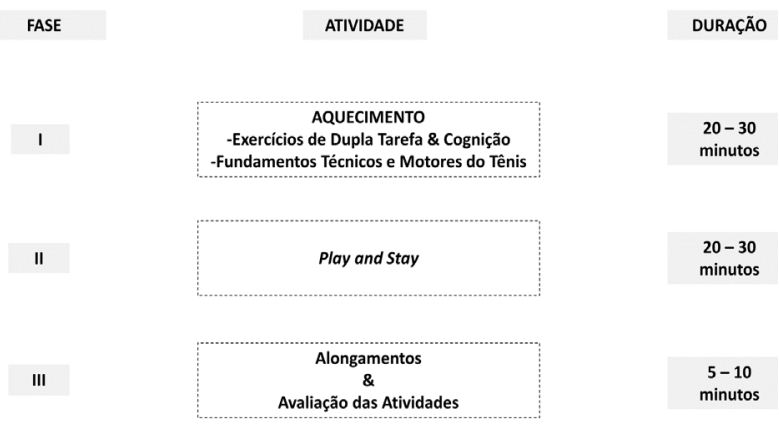

Figura 3. Estrutura da aula do programa "DT-Tênis 60+". Fonte: Autores, 2019.

A aula possui duração de 60 minutos e é estruturada em 3 momentos conforme apresentado na Figura 3. A primeira etapa da aula é destinada para o aquecimento e introdução das atividades de "dupla tarefa", compostas pela introdução dos fundamentos do tênis, juntamente com tarefas cognitivas (Figura 2). Considerando que o principal objetivo do programa de treinamento "DT-Tênis 60+" não é o desenvolvimento de uma técnica perfeita, mas sim melhorar as capacidades funcionais e cognitivas dos idosos à realização de suas AVDs, bem como, oferecer aos alunos idosos um espaço à troca de experiências e de lazer, sugere-se que os procedimentos evitem atividades analíticas e tecnicistas. Por essa razão, na segunda fase (Figura 3), utiliza-se a metodologia de ensino preconizada pelo programa Play and Stay (ITF, 2012).

Esta metodologia valoriza o ensino baseado no jogo contextualizado, utilizando quadras reduzidas, bolas com menos pressão e raquetes específicas para as diferentes faixas etárias (CORTELA et. al, 2012; LÉVI, 2013). Considera-se importante a utilização de raquetes de tamanho menor como forma de proteger o braço do idoso de possíveis lesões decorrentes do esforço repetitivo. Na medida em que ele se adapta ao esporte e desenvolva a musculatura do braço, passamos a utilizar raquetes de tamanho adulto. Outra adaptação utilizada para melhorar a 
troca de bolas e o jogo propriamente dito é a utilização de bolas plásticas (tipo "Dente de Leite"). Isto se justifica porque o uso desta bola aumenta substancialmente o tempo de permanência do idoso no exercício.

Ao final da aula é conduzida uma seção de alongamento dos principais grupos musculares utilizados (Figura 3). Após a sessão de alongamentos, é realizada uma avaliação com os alunos sobre o as atividades do dia, sobre as dificuldades enfrentadas e os avanços obtidos. Entendemos que este momento é fundamental para que o aluno idoso se sinta valorizado e continue participando das aulas.

\section{Exemplos práticos do programa de treinamento "DT-Tênis 60+"}

A Figura 4 introduz atividades próprias para adaptação do aluno idoso à bola e à raquete, em situações específicas do treinamento de prevenção em quedas. $O$ conjunto de materiais utilizados buscam adaptar o aluno à marcha em diferentes condições do solo, isso implica em: a) superfícies estáveis/instáveis, b) bases reduzidas, 3) transposição de obstáculos, e 4) desvio de obstáculos. Nas Imagens A e B são apresentados exercícios em que o aluno deve associar à marcha com o domínio da raquete e bola (opcional), em diferentes bases, sentidos e direções.

Enquanto que as Imagens C e D mostram exemplos de exercícios, que combinam o ensino dos fundamentos do Tênis com a simulação de situações de vida diária, como desviar de objetos e pessoas ou subir escadas ${ }^{1}$. O treinamento cognitivo pode ser executado durante todas as atividades. Para tanto, os alunos são submetidos à diferentes cargas de tarefas cognitivas, que estimulam o desenvolvimento das funções executivas. As tarefas cognitivas consistem em problemas aritméticos, retenção de memória e aprimoramento da fluência verbal. A técnica utilizada nos procedimentos metodológicos do treinamento cognitivo pode ser visualizada na Figura 2.

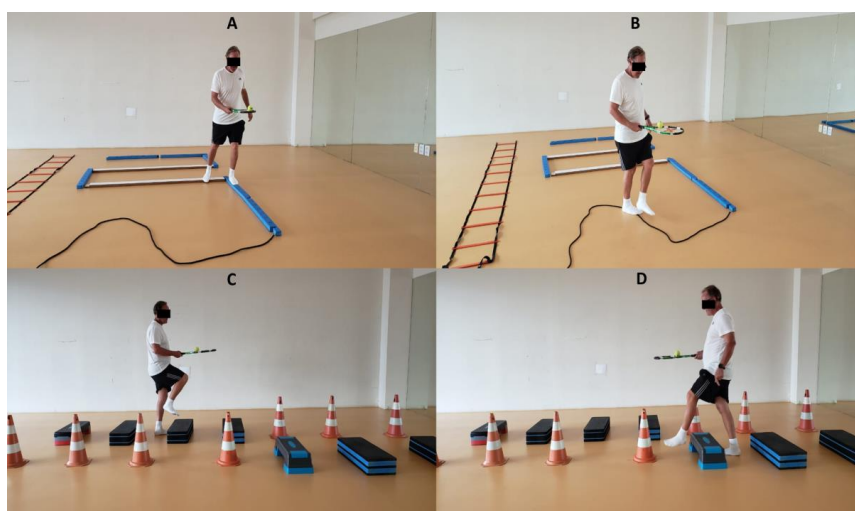

Figura 4. "DT-Tênis 60+", atividades para adaptação à bola e a raquete durante à marcha.

Fonte: Autores, 2019.

A Figura 5 apresenta um conjunto de atividades para o ensino do forehand, conforme o programa de treinamento "DT-Tê-

${ }^{1}$ Sugere-se que nesta fase do treinamento os alunos executem os exercícios descalços. A medida protege os idosos de possíveis acidentes (entorses) ao caminhar nas bases instáveis. Além disso, há estimulo dos proprioceptores localizados na regiáo plantar, responsáveis pelo envio via aferente de mulos posturas corretivos. 0 . snvelhecimento fisiológico também altera a sensibilidante da estiplantar, principalmente, da população diabética. Por essa razão, a prática dos exercícios descalço pode auxiliar o aluno idoso a restabelecer as funções sensitivas da região plantar, auxiliando significativamente à prevenção de quedas. nis $60+"$. Durante o processo de aprendizagem dos fundamentos técnicos e motores o aluno recebe constantemente cargas de tarefas, que buscam estimular sua capacidade cognitiva, memória e fluência verbal (Figura 2). É importante destacar que a execução dos fundamentos técnicos não tem por objetivo o desenvolvimento de uma técnica estereotipada ou idealizada a partir de um modelo de jogador pré-determinado, o que se busca é que o aluno possa internalizar e externar (Figura 1) o conjunto de gestos motores básicos próprios dos fundamentos técnicos.

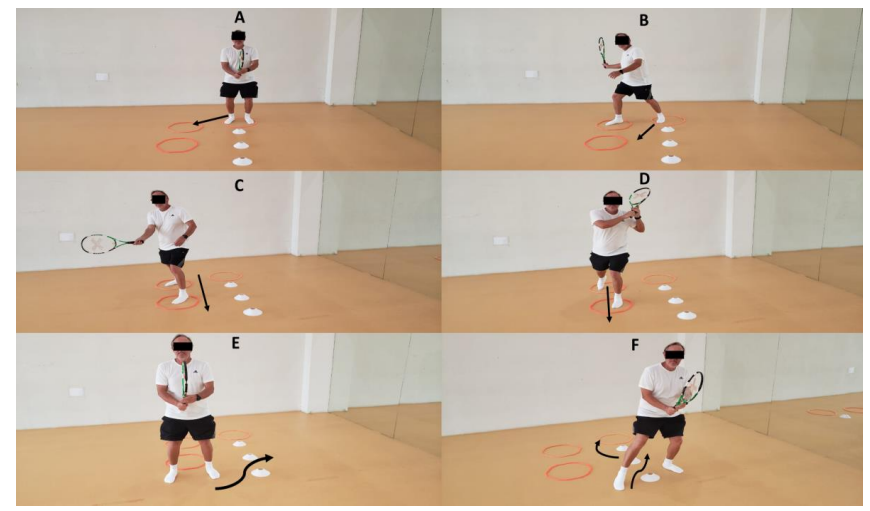

Figura 5. Programa "DT-Tênis 60+" exercícios para aprendizagem técnica do forehand Fonte: Autores, 2019

A Figura 5 apresenta seis sequências de uma proposta de exercícios para o aprendizado do forehand. Para que exista um melhor entendimento/aprendizado dos alunos sobre o modo correto de execução das tarefas motora, em combinação com as tarefas cognitivas, aconselhasse que: a) durante o aquecimento os gestos motores sejam introduzidos de forma livre e lúdica, b) a técnica das tarefas cognitivas do dia seja parcialmente apresentada aos alunos. Apesar da sequência de movimento (Figura 5) ter base discreta, início e fim determinado, e possuir características de uma habilidade fechada, ou seja, executada em ambiente previsível que permite o planejamento antecipado das ações (LAJOIE et al., 2016), ela é uma atividade de dupla tarefa porque combina informações motoras com cognitivas.

Aconselha-se também que as tarefas cognitivas realizadas durante o aquecimento não sejam repetidas durante o treinamento de dupla tarefa. Sugere-se, portanto, a manutenção da técnica seguida pela variação do grau de complexidade estabelecido para o estímulo cognitivo. A literatura especializada (LIEBHERR et al., 2016; GHAI; GHAl; EFFENBERG, 2017) sugere alguns exemplos/estratégias para o aumento da complexidade dos estímulos cognitivos:

\section{- Contagem progressiva de 1-10; \\ - Contagem regressiva de 10-1, 100-99;}

- Técnica de contagens de 2 em 2 ou de 3 em 3 números;

- Tarefas que envolvam dias da semana, meses do ano, nomes de animais (mamíferos, répteis, aves), nomes de flores, cidades, países, classes de alimentos;

- Uso de trava-línguas: este artifício é excelente para o desenvolvimento da habilidade verbal e de memória, além de estimular a capacidade cognitiva de forma lúdica. Trava-línguas criam desafios interessantes no treinamento "DT-Tênis 60+", pois obrigam o aluno a reproduzir, com clareza e rapidez, frases e versos que possuem dificuldade para a pronúncia. 
Por conseguinte, com o auxílio das estratégias de sistematização propostas para construção dos exercícios do programa do treinamento "DT-Tênis 60+" (Figura 2) professores de Tênis poderão criar novos exercícios, ampliando o leque de estratégias úteis para o aprendizado do forehand (Imagem A-F) com foco na prevenção de quedas.

\section{CONCLUSÃO}

O estudo apresentou a proposta conceitual para sistematização das atividades do programa de treinamento "DT-Tênis $60+"$. Para tanto, o texto destacou uma série de procedimentos úteis à estruturação de exercícios que facilitem e qualifiquem o ensino e a aprendizagem do tênis junto à população idosa. Outra questão trabalhada na proposta "DT-Tênis 60+" foi a prevenção de quedas, que é considerada na área do envelhecimento humano como questão de saúde pública. Os procedimentos do presente estudo partiram da revisão de literatura sobre os temas envelhecimento humano e queda de idosos, incluindo ainda as metodologias Play and Stay e Dual Task. A partir disso, foram criados modelos conceituais que auxiliam no entendimento sobre como os exercícios da proposta "DT-Tênis 60+" poderiam beneficiar as capacidades físicas, funcionais e cognitivas de idosos, reduzindo, consequentemente, o risco de quedas.

Considera-se que o presente estudo possui limitações. Embora os exercícios da proposta "DT-Tênis 60+" estejam sendo aplicados há 14 meses com idosos membros de uma Universidade Aberta à Terceira Idade, localizada na região nordeste do Brasil, e se tenha constatado melhoras significativas dos alunos em bateria de testes funcionais, avaliações do controle postural, velocidade da marcha e desempenho cognitivo, ainda não há um estudo publicado que comprove a efetividade das estratégias do treinamento "DT-Tênis $60+$ ". Portanto, até o momento, as informações apresentadas neste texto permanecem como um estudo teórico especulativo. Outra questão é que a literatura especializada da área do tênis e também do envelhecimento humano não oferecem informações sobre estudos que tenham investigado a relação entre o tênis, o idoso, a prevenção de quedas e o treinamento de dupla tarefa". Por essa razão, não foi possível tecer comparações entre a proposta "DT-Tênis 60+" e estudos anteriores.

Por outro lado, a ausência de estudos prévios pode ser interpretada como um ponto positivo para realização e divulgação da presente proposta de sistematização, uma vez que um dos princípios da "teoria do conhecimento" (epistemologia) consiste em incentivar a produção e o ganho de informações. Nessa perspectiva, espera-se que as informações apresentadas pelo presente estudo estimulem pesquisadores a desenvolverem futuros estudos, o que pode talvez criar um novo campo de investigação, associando o ensino e a prática do tênis com o processo de envelhecimento humano

\section{REFERÊNCIAS}

ABREU, D. R. DE O. M.; NOVAES, E. S.; OLIVEIRA, R. R. Internação e mortalidade por quedas em idosos no Brasil: análise de tendência. Ciência \& Saúde Coletiva, Rio de Janeiro, v. 23, n. 4, p. 1131-41, 2018.

BANIQUED, P. L.; GALLEN, C. L.; VOSS, M. W.; BURZYNSKA, A. Z.; WONG, C. N.; COOKE, G. E.; ... ; D'ESPOSITO, M. Brain network modularity predicts exercise-related executive function gains in older adults. Frontiers in Aging Neuroscience, New York, v. 9, p. 1-17, 2018.
BARROS, I. F. O. DE; PEREIRA, M. B.; WEILLER, T. H. Óbitos e Internações por Quedas em Idosos Brasileiros: Revisão Integrativa da Literatura. Revista Kairós Gerontologia, São Paulo, v. 19, n. 4, p. 363-82, 2016.

BAZELLO, B.; PORTELLA, F. C.; ANTUNES, G. P. P.; GATTI, A. L. Prática esportiva do idoso: autoimagem, autoestima e qualidade de vida. Boletim de Psicologia, São Paulo, v. 66, n. 145, p. 187-97, 2016

BOUAZIZ, W.; VOGEL, T.; SCHMITT, E.; KALTENBACH, G.; GENY, B.; LANG, P. $O$. Health benefits of aerobic training programs in adults aged 70 or over: asystematic review. Archives of Gerontology and Geriatrics, Amsterdam, v. 69 , p. $110-27,2016$

CACHIONI, M.; SATHLER, S.; BATISTONI, T. Bem-estar subjetivo e psicológico na velhice sob a perspectiva do conviver e do aprender. Kairós Gerontologia, São Paulo, v. 15, p. 9-22, 2012

CASERTA, R. J.; YOUNG, J.; JANELLE, C. M. Old dogs, new tricks: Training the perceptual skills of senior tennis players. Journal of Sport and Exercise Psychology, Bath, v. 29, n. 4, p. 479-97, 2007.

CORTELA, C. C.; FUENTES, J. P.; ABURACHID, L. M. C.; KIST, C.; CORTELA, D. N. R. Iniciação esportiva ao tênis de campo: um retrato do programa play and stay à luz da pedagogia do esporte. Conexões, Campinas, v. 10, n. 2, p. 214-34, 2012

CULPIN, S. Effects of long-term participation in tennis on cognitive function in elderly individuals. Joondalop: Edith Cowan University, 2018.

FASANO, A.; PLOTNIK, M.; BOVE, F.; BERARDELLI, A. The neurobiology of falls. Neurological Sciences, Milano, v. 33, n. 6, p. 1215-23, 2012.

FRANCO, M. A.; MENDES, M. I. B. de S. Fenomenologia e Educação Física: uma revisão dos conceitos de corpo e motricidade. Motrivivência, Florianópolis, v. 27, n. 45, p. 209-18, 2015

GHAI, S.; GHAI, I.; EFFENBERG, A. O. Effects of dual tasks and dual-task training on postural stability: a systematic review and meta-analysis. Clinical Interventions in Aging, Auckland, v. 12, p. 557-77, 2017.

GOUVEIA, B. R. : GOUVEIA, E. R.; IHLE, A. JARDIM, H. G.; MARTINS, M. M. FREITAS, D. L.; KLIEGEL, M. The effect of the probalance programme on health-related quality of life of community-dwelling older adults: a randomised controlled trial. Archives of Gerontology and Geriatrics, Amsterdam, v. 74, p. 26-31, 2018.

GROPPEL, J.; DINUBILE, N. Tennis: for the health of it! Physician and Sportsmedicine, Minneapolis, v. 37, n. 2, p. 40-50, 2009

HALL, C. D.; MISZKO, T.; WOLF, S. L. Effects of tai chi intervention on dual-task ability in older adults: a pilot study. Archives of Physical Medicine and Rehabilitation, Chicago, v. 90, n. 3, p. 525-9, 2009

HERNANDES, E. S. C.; BARROS, J. F. Efeitos de um programa de atividades físicas e educacionais para idosos sobre o desempenho em testes de atividades da vida diária. Revista Brasileira de Ciência e Movimento, Brasília, v. 12, n. 2, p. 43-50, 2004

ITF. International Tennis Federations. Play and Stay. Disponível em: $<$ http:// www.tennisplayandstay.com/home.aspx>. Acessado em: 21 de janeiro de 2020.

JUCHEM, L.; DA SILVA, E. B.; da SILVA, A. S. A prática do tênis no Programa Vida Ativa: relato de experiência. Extramuros, Petrolina, v. 3, n. 1, p. 27-37, 2016

KALACHE, A. Relatório global da OMS sobre prevenção de quedas na velhice. Who library cataloguing-in-publication data, Geneve, v. 1, n. 1, p. 1-64 2007.

KEARNEY, F. C.; HARWOOD, R. H.; GLADMAN, J. R. F. LINCOLN, N.; MASUD, T. The relationship between executive function and falls and gait abnormalities in older adults: a systematic review. Dementia and Geriatric Cognitive Disorders, Basel, v. 36, n. 1-2, p. 20-35, 2013.

KHANUJA, K.; JOKI, J.; BACHMANN, G.; CUCCURULLO, S. Gait and balance in the aging population: Fall prevention using innovation and technology. Maturitas, Limerick, v. 110, p. 51-6, 2018.

KLEINER, A.F.R ; DE CAMARGO, D. X: SCHLITTER S. A. M.; SÁNCHEZ-ARIAS, M. D-L. O papel dos sistemas visual, vestibular, somatosensorial e auditivo para o controle postural. Revista Neurociencias, São Paulo, v. 19, n. 2, p. 349-57, 2011

LAJOIE, Y.; RICHER, N.; JEHU, D. A.; TRAN, Y. Continuous cognitive tasks improve postural control compared to discrete cognitive tasks. Journal of Motor Behavior, Washington, v. 48, n. 3, p. 264-9, 2016

LAKATOS, E. M.; MARCONI, M. de A. Fundamentos da metodologia científica. 4. ed. São Paulo: Atlas, 2003.

LÉVI, F. Tennis play and stay for beginners aged 65 and over. ITF Coaching 
and Sport Science Review, London, v. 61, n. 21, p. 26-8. 2013

LIEBHERR, M.; SCHUBERT, P.; SCHIEBENER, J.; KERSTEN, S.; HASS, C. T. Dual-tasking and aging-About multiple perspectives and possible implementations in interventions for the elderly. Cogent Psychology, Zurich, v. 3, n. 1, p. 1-14, 2016

MARANESI, E.; MERLO, A; FIORETTI, S.; ZEMP, D. D.; CAMPANINI, I.; QUADRI, $P$. A statistical approach to discriminate between non-fallers, rare fallers and frequent fallers in older adults based on posturographic data. Clinical Biomechanics, Oxford, v. 32, p. 8-13, 2016.

McHUGH, D.; GIL, J. Senescence and aging: Causes, consequences, and therapeutic avenues. Journal of Cell Biology, New York, v. 217, n. 1, p. 65-77, 2018.

MCMULLAN, I. I.; MCDONOUGH S. M - TULLY, M. A. CUPPLES, M. CASSON, K.; BUNTING, B. P. The association between balance and free- living physical activity in an older community-dwelling adult population: a systematic review and meta-analysis. BMC public health, London, v. 18, n. 1, p. 431 2018.

MENANT, J. C.; SCHOENE, D.; SAROFIN, M.; LORD, S.R. Single and dual task tests of gait speed are equivalent in the prediction of falls in older people: systematic review and meta-analysis. Ageing Research Reviews, Oxford, v. 16 , n. 1, p. 83-104, 2014

MERLEAU-PONTY, M. Fenomenologia da Percepção. 2. ed. São Paulo: Martins Fontes, 1999.

MONTERO-ODASSO, M.; SPEECHLEY, M. Falls in cognitively impaired older adults: implications for risk assessment and prevention. Journal of the American Geriatrics Society, New York, v. 66, n. 2, p. 367-75, 2018.

MUELLER, J.; RODRIGUES, O. A. F. O tênis nas escolas: uma prática apropriada a cultura escolar. In: BALBINOTTI, C. (Org.). O ensino do tênis: novas perspectiva de aprendizagem. Porto Alegre: Artmed, 2009. p. 61-79.

NASCIMENTO, M. D. M. Benefits of the pilates method in preventing elderly falls. Journal of Physical Fitness, Medicine \& Treatment in Sports, Irvine, v. 4, n. 1, p. 1-3, 2018.

NASCIMENTO, M. de M. Fall in older adults: considerations on balance regulation, postural strategies, and physical exercise. Geriatrics, Gerontology and Aging, Rio de Janeiro, v. 13, n. 2, p. 1-8, 2019.

PLUIM, B. M.; STAAL, J. B.; MARKS, B. L.; MILLER, S.; MYLEY, D. Health benefits of tennis. British Journal of Sports Medicine, Loughborough, v. 41, n. 11, p. 760-8, 2007.

PLUMMER, P.; IYIGÜN, G. Effects of physical exercise interventions on dualtask gait speed following stroke: a systematic review and meta-analysis. Archives of Physical Medicine and Rehabilitation, Chicago, v. 99, n. 12, p. 2548-60, 2018.

RAFFEGEAU, T. E.; KREHBIEL, L. M. KANG, N.; THIJS, F. J. ALTMANN, L. J. P.; CAURAUGH, J. H.; HASS, C. H.. A meta-analysis: Parkinson's disease and dual-task walking. Parkinsonism and Related Disorders, Kidlington, v. 62, p. 28-35, 2019.

SHERRINGTON, C; MICHALEFF, Z. A.; FAIRHALL, N.; PAUL, S. S.; TIEDEMANN A.; WHITNEY, J.; ... ; LORD, S.R. Exercise to prevent falls in older adults: An updated systematic review and meta-analysis. British Journal of Sports Medicine, Loughborough, v. 51, n. 24, p. 1749-57, 2017.

SILSUPADOL, P.; SHUMWAY-COOK, A.; LUGADE, V.; VAN DONKELAAR, P. CHOU, L.S.; MAYR, U.; WOOLLACOTT, M. H. Effects of single-task versus dual-task training on balance performance in older adults: a double-blind randomized controlled. Archives of Physical Medicine and Rehabilitation Chicago, v. 90, n. 3, p. 381-7, 2009.

SILVA, M. C.; MARINS, E. F.; SPIEKER, C. V. Prática do tênis em idosos: estudo descritivo na cidade de Pelotas-RS, Brasil. Estudos Interdisciplinares sobre o Envelhecimento, Porto Alegre, v. 19, n. 1, p. 235-48, 2014.

SILVA, G. M. L. DA; CAMINHA, I. D. O.; GOMES, I. S. O corpo e o tempo: a percepção dos idosos de um grupo de convivência. Pensar a Prática, Goiânia, v. 16 , n. 4, p. 1081-97, 2013

SILVA, N. S. R.; DA ROCHA, F. W. R.; LIMA, M. O. M.; DE BARROS, S. L.; DE SOUZA, A. R.; NASCIMENTO, T. S. T. Percepção dos benefícios da hidroginástica pelo praticante quanto aos aspectos físicos, psíquicos e sociais em idosos da terceira idade. Revista Interfaces da Saúde, Aracati, v. 4, n. 1, p. 23-46, 2017.

SMITH, E.; CUSACK, T.; BLAKE, C. The effect of a dual task on gait speed in community dwelling older adults: A systematic review and meta-analysis. Gait and Posture, Oxford, v. 44, p. 250-8, 2016.

SOUZA, L. K.; COELHO, B. S; FREIRE, B.; DELEVATTI, R.; RONCADA, C.; TIG GEMANN, C.; DIAS, C. Comparação dos níveis de força e equilíbrio entre idosos praticantes de musculação e de hidroginástica. Revista Brasileira de
Atividade Física e Saúde, Londrina, v. 19, n. 5, p. 647-55, 2014.

SWANK, A. N. Physical dimensions of aging. Medicine \& Science in Sports \& Exercise, Madison, v. 28, n. 3, p. 399, 1996.

STINS, J. F.; SCHNEIDER, I. K.; KOOLE, S. L.; BEEK, P. J. The influence of motor imagery on postural sway: differential effects of type of body movement and person perspective. Advances in Cognitive Psychology, Warsaw, v. 11, n. 3, p. 77-83, 2015.

UEHARA, E.; CHARCHAT-FICHMAN, H.; LANDEIRA-FERNANDEZ, J. Funções executivas: um retrato integrativo dos principais modelos e teorias desse conceito. Revista Neuropsicologia Latinoamericana, Porto Alegre, v. 5, n. 3, p. 25-37, 2013

WOLLESEN, B.; SCHULTZ, S.; SEYDELL, L.; DELBAERE, K. Does dual task training improve walking performance of older adults with concern of falling? BMC Geriatrics, London, v. 17, n. 1, p. 1-9, 2017.

YOGEV-SELIGMANN, G.; HAUSDORFF, J. M.; GILADI, N. The role of executive function and attention in gait. Movement Disorders, New York, v. 23, n. 3, p. $329-42,2008$.

\section{AGRADECIMENTOS}

Os autores agradecem à Pró-Reitoria de Extensão da Universidade Federal do Vale do São Francisco (Univasf) pela viabilização do Projeto de Extensão "Iniciação ao Tênis: esporte e lazer para a comunidade de Petrolina (PE) e Juazeiro (BA)".

\section{CONFLITO DE INTERESSE}

Os autores do estudo declaram não haver conflito de interesses.

\section{FINANCIAMENTO}

Este estudo não teve apoio financeiro.

\section{ORCID E E-MAIL DOS AUTORES}

Luciano Juchem (Autor Correspondente) ORCID: 0000-0002-9713-0834.

E-mail: luciano.juchem@univasf.edu.br

Marcelo de Maio Nascimento ORCID: 0000-0002-3577-3439.

E-mail: marcelo.nascimento@univasf.edu.br 